\title{
A unified model of supernova driven by magnetic monopoles
}

\author{
Qiu-He Peng ${ }^{1}$, Jing-Jing, Liu ${ }^{2}$, and Chih-Kang \\ Chou $^{3}$
}

\begin{abstract}
In this paper, we first discuss a series of important but puzzling physical mechanisms concerning the energy source, various kinds of core collapsed supernovae explosion mechanisms during central gravitational collapse in astrophysics. We also discuss the puzzle of possible association of $\gamma$-ray burst with gravitational wave perturbation, the heat source for the molten interior of the core of the earth and finally the puzzling problem of the cooling of white dwarfs. We then make use of the estimations for the space flux of magnetic monopoles (hereafter MMs) and nucleon decay induced by MMs (called the Rubakov-Callen(RC) effect) to obtain the luminosity due to the RC effect. In terms of the formula for this RC luminosity, we present a unified treatment for the heat source of the Earth's core, the energy source for the white dwarf interior, various kinds of core collapsed supernovae (Type II Supernova (SNII), Type Ib Supernova (SNIb), Type Ic Supernova (SNIc), Super luminous supernova (SLSN)), and the production mechanism for $\gamma$-ray burst. This unified model can also be used to reasonably explain the possible association of the short $\gamma$-ray burst detected by the Fermi $\gamma$-ray Burst Monitoring Satellite (GBM) with the LIGO gravitational wave event GW150914 in September 2015 .
\end{abstract}

Keywords stars: supernovae, stars: evolution, Physical Date and Processes: nuclear reactions.

Qiu-He Peng, Jing-Jing, Liu , and Chih-Kang Chou

Corresponding to Jing-Jing, Liu. (liujingjing68@126.com)

${ }^{1}$ Department of Astronomy, Nanjing University, Nanjing, Jiangshu 210000, China.

${ }^{2}$ College of Marine Science and Technology, Hainan Tropical Ocean University, Sanya, Hainan 572022, China.

${ }^{3}$ National Astronomical Observatory, Chinese Academy of Sciences, Beijing, 100000, China.
1 The puzzle of the explosion mechanisms for the various kinds of core collapsed supernovae

Although the true reason of supernovae explosion due to gravitational collapse of the central core is not thoroughly understood so far, considerable insight has been obtained concerning both the physics of the gravitational collapse of the whole star after the end of thermal nuclear fusion for massive stars, and the initial stage of the collapse and the physical scenario why the entire star can not explode (Bethe et al. 1990; Woosley et al. 1986; Woosley 1991). The masses of the pre-main sequence supernova progenitor due to gravitational collapse of the central core may be roughly classified as follows: SNII, SN1b, SN1c (the physical origin for the long $\gamma$-ray burst) with messes respectively given by $M=(8-25) M_{\odot}, M=(30-60) M_{\odot}$ and $M=(80-150) M_{\odot}$. In addition, we also estimate the pre-main sequence mass for the brightest supernovae SLSN to be $M=(200-1000) M_{\odot}$ or more.

For the supernovae SNII and SNIb, the physical mechanism for the gravitational collapse of the stellar core is as follows. An iron core with high temperature $T_{c} \approx(3-5) \times 10^{9} \mathrm{~K}$ and high density $\rho_{c} \approx$ $(2-3) \times 10^{9} \mathrm{~g} / \mathrm{cm}^{3}$ is formed after the end of thermal nuclear fusion in the central core of massive stars. The election gas is in a highly relativistic degenerate state such that the Fermi energy of the election gas is obviously higher than the energy threshold for electron captured by iron nuclei, thereby causing large amount of the electrons quickly captured by the iron nuclei (and by the elements of the iron family) via the electron capture processes. Since large amount of free electrons quickly break into the nucleus the resulting electron pressure in the core is greatly reduced, and it then causes the entire iron core to a fast gravitational collapsing. In the outer region the speed of the collapse towards the center almost reaches half that of 
the free fall. In the inner region, however, it was generally believed that the core collapse was homogeneous as first suggested by Colgate et al. (1966). But in 2004, Peng et al. (2004) pointed out that since the electron capture process increases very fast as the density is increased, the resulting electron pressure decreases even faster with the radial distance towards the center. Consequently, the gravitational collapse in the central region should be in an accelerating state. Our recent research series showed that the weak interaction (e.g., electron capture and beta decay) plays a critical role in the process of supernova explosion (e.g., Liu (2013, 2014); Liu \& Gu (2016); Liu et al. (2016, 2017)).

General idea of the collapsed process of the core collapse of the supernova is following. The collapsing speed of matter at the interface of the inner and outer regions may reach as high as $(1 / 8-1 / 4) c$, where $c$ is the speed of light in vacuum. The central density of the star reaches $\rho_{c} \approx(2-5) \rho_{n u c}$ (where $\rho_{n u c}$ is the nuclear density), the supporting pressure against collapse becomes the non-relativistic degenerate neutron gas which is both dynamically and thermodynamically very stable (the non-relativistic degenerate neutron pressure is much higher than the degenerate pressure of the relativistic electron gas). The collapse of the inner region is quickly stopped. The non-relativistic and degenerate neutron gas not only resists the inward collapse of the matter with lower density in the outer region but also drive the matter with lower density in the outer region moving violently outwards with tremendous speeds and a so-called rebound shock with Mach number $(1-2)$ is then formed outside the interface between the inner and outer region. The rebound shock wave carries with it huge amount of energy (i.e., his energy is transformed from the gravitational energy released by the matter in the core during the collapse, it may reach $\left.10^{52}-10^{53} \mathrm{ergs}\right)$. The temperature of rebound shock front may reach as high as $10^{11} \mathrm{~K}$. At such temperatures, large number of the thermal $\gamma$-ray photons may have energy about $10 \mathrm{Mev}$ and these energetic thermal $\gamma$ ray photons immediately break the iron nucleus (and the elements of iron family) into nucleons and particles: ${ }^{56} \mathrm{Fe}+\gamma \rightarrow 13 \alpha+4 \mathrm{n}, \alpha+\gamma \rightarrow 2 p+2 n$.

Since this process consumes huge amount of energy and all reliable stellar evolution models predict a rather large mass for the outer region of the iron core thereby causing the energy of the rebound shock wave completely depleted before it can break and completely destroy the outer region of the collapsing star. In other words, it is impossible to make the whole outer region of the star to explode outwards and this "instantaneous explosion mechanism" for supernovae explosion fails. If we adopt the idea the of accelerated collapse of the central core as proposed by Peng et al. (2004) which is due to the increase of the electron capture rates with density at the supernovae core, the mass of the inner core during the initial stage of the collapse clearly decreases. Along this line of thought, we also make use of the simplest model for gravitational collapse with spherical symmetry and some artificial parameter to simulate the collapse, The supernovae explosion is possible for some of our models with appropriate parameters (Luo et al. 2010).

Because the failure of the instantaneous explosion mechanism Wilson proposed a neutrino delayed mechanism for supernovae explosion (Wilson et al. 1988). After the collapse of the supernovae core a nascent neutron star with high temperature $10^{11} \mathrm{~K}$ is formed. Wilson proposed that a strong neutrino flux will be produced from the nascent neutron star. The weak interaction between the strong neutrino flux with the matter of outer region of the supernovae can lead to explosion of the outer region and forming neutrino explosion. However, two questions are open for the neutrino delayed mechanism: a) How this strong neutrino flux is generated? b) Whether the supernova can be expelled by the weak interaction between the strong neutrino flux with matter in the outer region of the supernova?

Based on high temperature, $10^{11} \mathrm{~K}$, of a nascent neutron star from the collapse of the supernovae core, we have proposed that in the high temperature interiors of the nascent neutron stars by supernovae core collapse, large amounts of neutrino flux with energy $10 \mathrm{Mev}$ maybe produced in less than $10^{-6}$ second via the phase transition processes (i.e. $u+e \rightarrow d+\nu_{e}, u+e \rightarrow s+\nu_{e}$, $u+d \rightarrow u+s)$ from the two flavor quarks $(\mathrm{u}, \mathrm{d})$ into the there flavor quarks (s, u, d) with total energy about $10^{52}$ erg (Dai et al. 1995). Nevertheless, it is still open question up to now whether the interaction between strong neutrino flux with matter can really generate the strong outward pressure to achieve supernovae explosion with outward initial speed $10^{4} \mathrm{~km} / \mathrm{s}$ and total kinetic energy $10^{49}$ ergs? Although considerable efforts have been devoted to study neutrino transport process and neutrino fluid dynamics with complicate (2D and 3D) numerical simulation, unfortunately supernovae explosion through self consistent theory and simulation has not been achieved. The representative reference in this regard may be illustrated by Buras et al. (2003), and Liebendoerfer et al. (1992).

It is generally believed that the progenitor of Ic type supernovae (SNIc) is the massive pre-main sequence with large mass $M=(80-150) M_{\odot}$ and the progenitor of the brightest supernovae (SLSN) is the pre-main sequence star with even large mass $\left(M>(200-500) M_{\odot}\right.$ ). These supermassive stars have already, lived through the stages of hydrogen burning (main sequence star) 
and helium burning (red giant star). Since the reaction rate of ${ }^{12} \mathrm{C}+\alpha \rightarrow{ }^{16} \mathrm{O}+\gamma$ is very fast, the abundance of ${ }^{12} \mathrm{C}$ in the core after helium burning may be lower than $8 \%$. The small amount of ${ }^{12} \mathrm{C}$ may continue to burn during the core collapse. The central region of the star then start to burn oxygen directly and the temperature of the core may reach $5 \times 10^{9}-10^{10} \mathrm{~K}$. At the high temperature region, a large number of high energy thermal $\gamma$-ray photons annihilate into election position pairs and the inverse reaction $\left(\gamma+\gamma \rightarrow e^{+}+e^{-}\right)$may reach thermal equilibrium. Meanwhile, large number of the selection positron pairs may annihilate into neutrino pairs at very fast rate and these energy outgoing neutrinos immediately escape from the star.

The energy carried away by the neutrinos is lost from the star and lead to the fast reduction of the interior thermal pressure and finally resulting in drastic gravitational collapse of the supernovae lore. This is well known as the instability generated by electron positron pair annihilation into neutrino pairs as proposed by Chiu \& Morrison (1960). This is the physical mechanism for the gravitational collapse of super-massive stars. From some astrophysical observational evidence it is generally that long $\gamma$-ray burst is associated with type Ic supernovae explosion. The explosion mechanism for SNIc has been investigated in terms numerical simulation since 2007. No critical progress has been achieved. Thus, the explosion mechanism for SNIc is still a puzzle.

As for the super luminous supernova (SLSN), the absolute magnitude of their peak luminosity is $M_{\text {peak }} \leq$ $-21^{m}$ (but the peak luminosity of the supernova usually is $M_{\text {peak }} \geq-20^{m}$ ). The ultra luminous supernova ASASSN-15lh discovered in Sep. 2015 (Dong et al. 2016), after 15 days from the peak luminosity, its luminosity is still up to $2.2 \times 10^{45} \mathrm{erg} / \mathrm{s}$. It is twice as bright as the brightest supernova luminosity up to date. Its radiation energy is up to $1.2 \times 10^{45}$ ergs during four months. Recently, the discussion about it is quite warm. For example, Dai et al. (2016) roughly guess the ultra bright supernova ASASSN-15lh might being the evidence for the existence of quark stars. However, this paper does not involve the discussion of the mechanism of the supernova explosion. The ASASSN-15lh phenomenon is still a doubt. In short, the mechanism of various types of supernova explosion is still a major mystery.

The plan of this paper is as follows. In Section 2, we introduce a new insight into MMs and their properties. In Section 3, we discuss astrophysical evidence for the existence of MMs. We introduce our model of quasars and AGNs containing MMs proposed in 1985 (Peng et al. 1985a, b) and predictions of the model pro- posed in Peng et al. (2001). It seems that the subsequent observational evidence favorably support our early predictions and the observed astrophysical evidences support the existence of MMs. We detailed discuss the models of the compelling mechanism for the various types of supernovae explosion in Section 4. In section 5, we investigate the other related puzzle of our model of supernova explosion driven by magnetic monopoles. For instance, the energy source for the hot molten interior of the earth's core and the puzzle of cooling of the white dwarfs are discussed. The collision and merge of two massive neutron stars containing MMs are also used to explain the possible association of the short $\gamma$-ray burst with the LIGO gravitational wave perturbation event GW150914 in Section 5. Finally some conclusions and outlooks are given in Section 6.

\section{Study on the Magnetic Monopole}

\subsection{Rubakov-Callen(RC) effect about Nucleon Decay induced by $\mathrm{MM}$}

During the period between 1970 and 1980 of the last century, particle physicists are especially interested in discussing the problem of MMs (Polvakov et al. 1974; Ma \& Tang. 1983; Shnir. 2005; 't Hooft. 1974). It is generally believed that super-massive MMs probably exist. In that times its mass was estimated as $m_{m} \sim$ $10^{16} m_{p}$, where $m_{p}$ is the mass of the proton. But it is estimated as $m_{m} \sim\left(10^{3}-10^{4}\right) m_{p}$ in recent years (Aab et al. 2016).

The magnetic charge of a MM is $g_{m}=3 h c /(2 e)=$ $0.988 \times 10^{-7}$ (c.g.s). As for possible applications to astrophysics, the most important property of MMs is that these exotic particles can induce nucleon decay, namely

$p M \rightarrow e^{+} \pi^{0} M+$ debris $\quad(85 \%)$,

$p M \rightarrow e^{+} \mu^{ \pm} M+$ debris $\quad(15 \%)$,

where pM means that a proton ( $\mathrm{p}$ ) catalyzed by magnetic monopoles $(\mathrm{M})$ decays into a positron and a neutral pi meson $\left(\pi^{0}\right)$ or decays into a positron and a pair of $\mu^{ \pm}$.

These MM induced reactions are proposed independently by Rubakov (1981) and Callan et al. (1983) with reaction cross section $\sigma=\left(10^{-25}-10^{-26}\right) \mathrm{cm}^{2}$. On the other hand, a much lower cross section $\sigma \approx$ $\left(10^{-36}\right) \mathrm{cm}^{2}$ is proposed by Wilczek. (1982). For simplicity, the reaction of MM induced nucleon decay is called the $\mathrm{RC}$ effect with the estimated typical cross section $\sigma \approx\left(10^{-30}\right) \mathrm{cm}^{2}$ or $\sigma \approx\left(10^{-30 \pm 6}\right) \mathrm{cm}^{2}$. 
2.2 The amount of MMs in space

It is generally believed that during the phase transitions in the very early universe, small amounts of MMs may be generated by the drastic oscillation and thermal agitation of the Higgs field . The amount of MMs may by defined as the ratio of the number of MMs, $N_{m}$, to that of the baryons, namely, $\xi=N_{\mathrm{M}} / N_{\mathrm{B}}$, where $N_{B}$ is the number of baryons. Here we define a parameter $\zeta_{s}$,named Newton saturation content, when the Newtonian gravity of a MM from the center of the stars, balance with the magnetic Coulomb repulsion by the interior total magnetic charge (with the same polarity). Newton saturation content is the ratio of the maximum number of MMs at the center of the stars and the number of baryons in accumulation area of the MM. Thus, the Newton saturation value is (Peng et al. 1985a)

$\zeta_{s}=G m_{\mathrm{B}} m_{m} / g_{m}^{2} \approx 1.9 \times 10^{-32} \frac{m_{m}}{10^{9} m_{p}}$,

where we take $10^{9} m_{p}$ as the mass of the magnetic monopole, which is intermediate between $10^{4} m_{p}$ Aab et al. 2016) and $10^{16} m_{p}$ (Polvakov et al. 1974; 't Hooft. 1974).

\subsection{The amounts of MMs contained in astronomical bodies}

The following discussions and predictions have been presented in our paper in 1985 (Peng et al. 1985a, b)

Firstly, in the early universe matters were in the ionized plasma state with very high temperature. The strong electromagnetic interaction between the MMs with plasma may lead to the existence of considerable amount of the MMs in the central region of astronomical bodies. This is because the formation of quasars and active galactic nuclei (AGNS) from huge and supermassive primordial cloud through gravitational collapse of the plasma via Jeans instability. Thus, the central region of our Milky Way galaxy, quasars and ANGS may contain more MMs with the Newtonian saturation value $\zeta_{s}$. in section 3 , we will discuss in more detail of our model for super-massive stellar objects with MMs . This model can provide huge amount of radiation and we may use it to replace the usual black hole model as the energy source.

Secondly, generally speaking, stars and planets are formed in the massive neutral hydrogen cloud via Jeans gravitational instability. The interaction between MMs with neutral hydrogen atoms is very weak so that there are very few of the MMs can fall towards the central region following gravitational collapse of the neutral hydrogen cloud and they are neglected. But some MMs may be contained in the interiors of stars and planets now. These MMs are mainly captured from space during their life time after their formation. The flux of the MMs captured from space may be estimated as follows (Peng et al. 1985a)

$$
\begin{gathered}
\phi_{m}=n_{m} \nu_{m}=10^{-4} c \zeta_{m}^{0} n_{\mathrm{B}}^{0}\left(\frac{\nu_{m}}{10^{-4} c}\right) \\
=6 \times 10^{-12}\left(\frac{\zeta_{m}^{0}}{10^{-20}}\right)\left(\frac{m_{m}}{10^{9} m_{p}}\right)^{-1}\left(\frac{n_{\mathrm{B}}^{0}}{1 \mathrm{~cm}^{-3}}\right)\left(\frac{\nu_{m}}{10^{-4} c}\right) \mathrm{cm}^{-2} \mathrm{~s}^{-1},
\end{gathered}
$$

where the superscript (0) denotes for the space, and $n_{\mathrm{B}}^{0}$ denotes the number density of baryons in the interstellar space of the Milky Way galaxy, $\nu_{m}$ represents the velocity of the MMs in space, and $\mathrm{c}$ is the speed of light. We use $\zeta_{m}^{0}$ to denote the value $\zeta_{m} \equiv\left(N_{m} / N_{B}\right)$ of MMs contained in space according to the upper limit given by Parker (1970), $\xi_{m}^{0} \leq 10^{-20}$. The Newtonian saturation value, $\xi_{s}$, is lower than the upper limit given by Parker by five order of magnitude $\left(\xi_{m}^{0} / \xi_{s} \leq 10^{5}\right)$.

The total number of MMs captured from space by stars or planets after their formation may be estimated o be (Peng et al. 1985a)

$$
\begin{aligned}
N_{m}=4 \pi R^{2} \phi_{m} t=5.0 \times 10^{28}\left(\frac{\zeta_{m}^{0}}{10^{-20}}\right)\left(\frac{n_{\mathrm{B}}^{0}}{1 \mathrm{~cm}^{-3}}\right)\left(\frac{m_{m}}{10^{9} m_{p}}\right)^{-1} \\
\times\left(\frac{\nu_{m}}{10^{-4} c}\right)\left(\frac{R}{R_{\odot}}\right)^{2}\left(\frac{t}{4.5 \times 10^{9} \mathrm{Yr}}\right) \mathrm{cm}^{-2} \mathrm{~S}^{-1}
\end{aligned}
$$

where $\mathrm{R}$ denotes the radius of the star and $t$ is the life time of the star. Thus, the MMs captured from interstellar space by stars and planets during their life time is proportional to the surface area and the life time of the stars and planets. The time scale of the stars in the main sequence stage is about $90 \%$ of the life time for the stars. Due to hydrogen burning in the main sequence stage of the stars the stars is relatively stable and the stellar radius changes little during the main sequence stage. However, the stellar radius during the red giant stage of the stars will increases up to more than 10 times of the radius of the main sequence stars at least, although the time scale of the stars in the red giant stage is only about $10 \%$ of the life time for the stars. Thus, the total number of MMs captured from space by the progenitor of supernova in its life time is about 10 times of one calculated by the formula (4) for this progenitor during its main sequence stage.

Since MMs are super heavy $\left(m_{m}>10^{3} m_{p}\right.$ at least $)$ so that the captured MMs accumulate in the stellar core with concentration $n_{m}=\zeta_{s} n_{B}$. The radius of the core $r_{c} \approx\left(N_{m} / \xi_{s} n_{\mathrm{B}}^{c}\right)^{1 / 3}$, where $n_{B}^{c}$ represents the average number density of the nucleons in the central core. The total mass of the MMs accumulated in the central region becomes only

$M_{m}=N_{m} m_{m} \approx 10^{-8} N_{m} \frac{m_{m}}{10^{16} m_{p}} \quad \mathrm{gm}$, 
Although this mass of the MMs accumulated in the central core is only a very small fraction of the total mass of the star, it can possibly provide huge luminosity (called RC luminosity) determined by the central density of the stars via the RC effect (nucleon decay induced by MMs)

\subsection{The Reason why MMs were not detected by Geophysical Laboratories}

Most physicists are very disappointed by the fact that MMs were not detected in the geophysical laboratories. Recently, the book from Wikimedia collected all the physics experiments designed for the detection of MMs and pointed out that all physics experiments fail to detect the existence of MMs until now. They also give the upper limit for the ratio of the number of MMs to that of the nucleons as $10^{-29}$. All these experiments are carried out either on the surface of the earth or in the earth's outer layer. Besides, in 1969 NASA sent the satellite explorer 35 (orbiting the moon) with GSFC magnetometer. Their scientific goal is to measure the difference between the magnetic north pole and the magnetic south pole of the moon so as to measure the number of MMs contained in the moon.

According to the measurements of Schatten (1970), and Schatten et al. (1983), they gave the upper limit for the ratio of the number of MMs to that of the nucleons as $6 \times 10^{-23}$. In order to carry out the detection experiments of MMs in the geophysics laboratories we may rewrite Equ. (3) as

$\phi_{m} \approx 0.25\left(\frac{\zeta_{m}^{0}}{10^{-20}}\right)\left(\frac{m_{m}}{10^{9} m_{p}}\right)^{-1}\left(\frac{n_{\mathrm{B}}^{0}}{1 \mathrm{~cm}^{-3}}\right)\left(\frac{\nu_{m}}{10^{-4} c}\right) m^{-2} \mathrm{Yr}^{-1}$

The expression (7) implies that the flux of the MMs in flight in interstellar space is very small. This means that the detection probability of captured magnetic monopoles in flight is only about one in 4 years even for large detector area $1 \mathrm{~m}^{2}$. This is the reason why MMs have not yet been detected by cosmic ray detector experiments and geophysical laboratories. The number of MMs captured by the earth after its formation (the age of the Earth is about 45 hundred million years) according to Equ.(5) is given by

$N_{m} \approx 5.0 \times 10^{24}\left(\frac{\zeta_{m}^{0}}{10^{-20}}\right)\left(\frac{m_{m}}{10^{9} m_{p}}\right)^{-1}\left(\frac{n_{\mathrm{B}}^{0}}{1 \mathrm{~cm}^{-3}}\right)\left(\frac{\nu_{m}}{10^{-4} c}\right)$

These super-heavy MMs all accumulate in the central region of the earth with radius less than $1 \mathrm{Km}$.
There are no MM in the mantle, the outer layer or the surface of the Earth. All experiments designed to detect MMs in the outer layer of the Earth are useless. Consequently, there are no MM in the outer crust of the earth.

The MMs captured by the moon is far lower than that captured by the earth because much larger surface area of the earth. The radius of the moon is about $1 / 4$ that of the earth and the age of the moon is about the same as that of the Earth. The number of MMs accumulated in the core of the moon may then be estimated to be

$$
N_{m} \approx 6.2 \times 10^{22}\left(\frac{\zeta_{m}^{0}}{10^{-20}}\right)\left(\frac{m_{m}}{10^{9} m_{p}}\right)^{-1}\left(\frac{n_{\mathrm{B}}^{0}}{1 \mathrm{~cm}^{-3}}\right)\left(\frac{\nu_{m}}{10^{-4} c}\right)
$$

where the mass of the moon is about $1 / 81$ that of the earth, i.e. $7.4 \times 10^{25} \mathrm{gm}$, thus, the ratio of the number of MMs to that of the nucleons for the moon is

$\zeta \approx 1.4 \times 10^{-27}\left(\frac{\zeta_{m}^{0}}{10^{-20}}\right)\left(\frac{m_{m}}{10^{9} m_{p}}\right)^{-1}\left(\frac{n_{\mathrm{B}}^{0}}{1 \mathrm{~cm}^{-3}}\right)\left(\frac{\nu_{m}}{10^{-4} c}\right)$.

The upper limit of the number of MMs contained in the Moon by the 1983 moon orbiting satellite experiments is $6 \times 10^{-33}$. This observed upper limit just gives a restrictive condition, which is given by

$\left(\frac{\zeta_{m}^{0}}{10^{-20}}\right)\left(\frac{m_{m}}{10^{9} m_{p}}\right)^{-1}\left(\frac{n_{\mathrm{B}}^{0}}{1 \mathrm{~cm}^{-3}}\right)\left(\frac{\nu_{m}}{10^{-4} c}\right) \leq 4 \times 10^{-6}$.

We anticipate that future satellite experiments orbiting the Earth or the Moon with more accurate GSFC magnetometer with improve measurement by $(3 \sim 4)$ orders of magnitude, so that the possible existence of MMs in the center of the Moon or the Earth may be determined. At least, Eq.(11) may provide a very good constraint.

Although geophysical laboratories can not determine the possible existence of MMs contained in the earth or the moon, most recent discovery in astrophysical observations bring some hints for the possible existence of MMs in the core of huge massive stellar object (see Section 3). This is because strong radial magnetic field with strength $>8 \mathrm{MG}$ was discovered in the vicinity $0.12 \mathrm{pc}$ of the central region of our Milky way Galaxy in 2013. The details of the connection between the discovery of strong radial magnetic field and the existence of magnetic monopoles will be elaborated in Section 3 . We would like to present a unified treatment for energy sources that may explain the explosion mechanisms for the various types of supernova explosions (including $\gamma$ ray bursts). We also may explain the phenomenon of 
the molten core for the Earth, cooling problem of white dwarfs (WDs). It will be shown that MMs located in the central region of the stellar objects may play the key role in our theory.

\subsection{The discussions of RC luminosity}

The total luminosity generated by the nucleon decay induced by MMs in the central region of various stellar objects may the estimated as follows

$L_{m} \approx \frac{4 \pi}{3} r_{c}^{3} n_{m} n_{\mathrm{B}}\left\langle\sigma \nu_{\mathrm{T}}\right\rangle m_{\mathrm{B}} c^{2}=N_{m} n_{B}\left\langle\sigma \nu_{\mathrm{T}}\right\rangle m_{\mathrm{B}} c^{2}$,

where $r_{c}$ is the radius of the stellar central region where MMs accumulate, $n_{m}, n_{B}$ are respectively the number density of MMs and nucleons, $N_{m}$ is the total number of MMs in the core of the stellar object and it is also the number of captured MMs since the birth of the stellar object. In the Equ.(12), $\sigma$ denotes the reaction cross section for the RC effect, $\sigma \approx$ $\left(10^{-25}-10^{-26}\right) \mathrm{cm}^{2}$, and $\nu=\nu_{\mathrm{T}}$ represents the thermal velocity of the nucleons relative to the MM . This is because MMs are super-heavy and their thermal velocities can be neglected. Consequently, we consider only the thermal velocity of the nucleon with $\nu_{\mathrm{T}}=\sqrt{2 k T / m_{\mathrm{B}}}$, where $\mathrm{T}$ denotes the temperature and $\mathrm{k}$ is Boltzmann's constant. $\left\langle\sigma \nu_{\mathrm{T}}\right\rangle$ depicts the thermal average.

In the RC process MMs induced nucleon decay, followed by nucleon decay into $\pi^{0}$ meson, $\mu^{ \pm}$leptons and positions $e^{+}, \mu^{ \pm}$and $\pi^{0}$ again decay into photons rand election position pairs $e^{ \pm}$. The positions then annihilate with the elections to photons. The net effect is that the rest mass energy of nucleons ( $m_{\mathrm{B}} c^{2}$ ) entirely converted to radiation energy with $100 \%$ efficiency. $\left(1 m_{\mathrm{B}} c^{2} \approx 1 \mathrm{GeV} \approx 10^{-3} \mathrm{ergs}\right)$.

\section{Astrophysical evidence for the existence of MMs}

Since 1985 we have seriously interested in studying the following problem: if the idea of MMs and the RC effect in particle physics are correct, what are their important effects on theoretical and observational astrophysics? (Peng et al. 1985a, b; Wang et al. 1985)

\subsection{Model of Quasars and AGN with MMs}

It is generally believed that the super-massive central regions of quasar and AGNs are formed from the gravitational collapse of primordial super-massive stellar clouds during the early epochs of the universe via Jeans instability.

During the early epoch of the universe the temperature is so high that the primordial stellar clouds are in the plasma state. Since the electromagnetic interaction of the MMs with the stellar plasma clouds is very strong such that large amounts of the MMs fall and accumulate in the central region of the stellar clouds following gravitational collapse via Jeans instability. These superheavy MMs accumulate in the central region as much as possible until the Newtonian saturation value $\left(\zeta_{s}\right)$ is reached. Using the $\mathrm{RC}$ effect as the energy source we proposed the models for quasars and AGNs with MMs 32 years ago (Peng et al. 1985a; Wang et al. 1985). The idea of our approach is as follows

First, we use MMs induced nucleon decay in to leptons as the main energy source of quasars and AGNs to replace the black hole model (the accretion flow may be used as a secondary source of energy) Second, the gravitational effect of the super-massive star containing MMs is similar to that of a black hole. The gravitational effects is almost the same for these two models outside $50 \mathrm{AU}$ from the center. The matter in the massive central core is relatively rarefied. The radio waves and infrared waves observed from the direction of the Galactic Center (hereafter the GC) in recent years are probably originated from the inner region of the supermassive star at the GC .

We note that super-massive stellar object with sufficient MMs have neither black hole horizon nor central singularity. This is because the reaction rate of the $\mathrm{RC}$ effect (nucleon decay into leptons induced by MMs) is proportional to the square of the matter density so that the central density cannot become infinite. Making use of the RC effect in particle physics we can avoid the problem of the central singularity due to the black hole of classical general relativity. In this way the theory of the physics involved is self consistent and harmonious.

\subsection{Main Predictions of our model and the observational test}

In 2001, we put forward five theoretical predictions for a model of a supper massive object with MMs in the GC (Peng et al. 2001). The main ideas are as follows

Large amount of positions, for our first prediction, are produced at the $\mathrm{GC}$ with production rates $6.4 \times 10^{42} e^{+} \mathrm{s}^{-1}$. It is in agreement with the high energy astrophysical observations $\left((3.4-6.3) \times 10^{42} e^{+} \mathrm{s}^{-1}\right)$ in 2003 by detecting the very strong spectral lines of election - position annihilation observed along the direction of the GC (Knödlseder 2003).

After the publication of this observational result, some theoretical models to explain both the $511 \mathrm{keV}$ 
and the GeV Gamma-rays from the direction of the GC appeared. For instance, during the period 2005 - 2006, Wang et al. proposed that there may be millisecond pulsars exist in the galactic center (e.g., Wang et al. (2005, 2006)). Boehm (2004) considered a dark matter model. Casse et al. (2004) assumed that there may exist $\gamma$-ray bursts in the GC. Cheng et al. (2007) proposed that black hole explosion activity may be used to explain the $511 \mathrm{keV}$ radiation. Our predications were published (Wang et al. 1985) and reemphasized in our paper (Peng et al. 2001). We would like to emphasized again that our predication for the production of large amount of positrons from the GC is quantitatively verified by the observations in 2003 (Knödlseder 2003).

The second prediction is that some strong high energy radiation with energy higher than $0.511 \mathrm{Mev}$ is simultaneously emitted from the huge massive stars with MMs at the GC. Their integral total energy is much higher than that for the election - position annihilation lines and much higher than the thermal luminosity of the central stellar object. This is also in agreement with observations.

The third prediction is that the MMs accumulated at the center of a supermassive stellar object can generate powerful radial magnetic field. The magnetic field strength is about $(20-100)$ Gauss at the stellar surface of radius 50 a.u. (Peng et al. 2001). This prediction is also in good agreement with the observation in 2013 (Eatough et al. 2013). Since the field strength of the radial magnetic field is inversely proportional to the square of the radial distance so that $B \approx(10-50) \mathrm{mG}$ at $r=0.12 \mathrm{pc} \approx 3.1 \times 10^{17} \mathrm{~cm}$. This prediction is also in good agreement with the lower limit $8 \mathrm{mG}$ determined from the observations in 2013 (Eatough et al. 2013).

In 2001, we pointed out that the prediction for the presence of radial magnetic field in the vicinity of the super-massive object at the GC can be tested in the not distant future by astrophysical observations (Peng et al. 2001). If powerful radial magnetic field is really confirmed by astrophysical observations, then our model is unique in the sense that our model can naturally predict the existence of strong radial magnetic field.

Fourthly, extreme high energy cosmic rays with energy $\left(10^{18}-10^{21}\right) \mathrm{eV}$ have been suggested to originate from accelerations in the AGN core region, jet shear layers (Letessier \& Stanev 2011). but if we assume that all super-massive galactic nuclei of the AGNs within the range of $50 \mathrm{Mpc}$ from the Earth contain saturated MMs (see the details in paper of Peng et al. (2002)). Of cause, there are many possibilities of the source of extreme high energy cosmic rays with energy in theory. For example, the review by Letessier \& Stanev (2011) mentions conventional models such as accelerations in the AGN core region, jet shear layers etc., and also several exotic models. Indeed, these models have their respectively issues, but in general, they should be considered as reasonable theoretical models.

Finally, we predicted the surface temperature of the super-massive stellar object at the GC to be $123 \mathrm{~K}$ and the corresponding peak value of the thermal radiation is roughly $10^{13} \mathrm{~Hz}$ (at the sub-millimeter range) (Peng et al. 2001), and this is quite close to the observed value of $10^{12} \mathrm{~Hz}$ (Falcke et al. 2013).

Based on the above predictions as well as the new discovery and the latest progress made in recent years, especially the discovery of the unusually strong radial magnetic field near the GC in 2013, we (see Peng et al. (2016a, b. c) pointed out that such powerful radial magnetic field necessarily stop the plasma in the accretion disk surrounding the GC from entering the inner core consequently, the large amount of radiation (radio, infrared and X-ray ) observed from the direction of the GC can not be generated by the accreting material. From this we assert that the black hole model of the GC of our Milk Way that has been prevalent for almost half a century must not be real. Our model of the super-massive star containing MMs for the GC is a useful alternative model that can explain the observed radiation from the GC. Moreover, in the Refs. Peng et al. (2016a, b c), we have shown that the observed strong radial magnetic field near the GC cannot be generated by the most effective mechanism (producing the magnetic field) known so far, e.g. the $\alpha$ dynamo and by all other mechanisms (producing the magnetic field) proposed in recent years. In addition, the observed powerful radial magnetic field at $r=0.12 \mathrm{pc}$ from the GC with magnetic field strength $B>\mathrm{mG}$ (Eatough et al. 2013) is almost the same as our prediction (at $r=0.12 \mathrm{pc}, \mathrm{B}=(10-50) \mathrm{mG}$ based on the model of super-massive object containing MMs at the GC.

The above three predictions (the first, third, and last one) are all completely independent. These predictions are all consistent with observations. These predictions are all consistent with observations. These predictions cannot be accidental coincidence. The discovery of powerful radial magnetic field near the GC may have the following two important physical significance: 1) the fact that unusually strong radial magnetic fields are discovered near the GC may be the convincing astrophysical evidence for the existence of MMs. Thus our model for quasars and AGNs containing MMs is reasonable. 2) The radiation originate from the direction of the GC cannot be generated by the standard model of the black hole with its accretion disk. 
3.3 On the MMs captured by stars and planets

Stars and planets are formed from the gravitational collapse of massive neutral hydrogen cloud. Since the interaction of MMs with the neutral hydrogen cloud is very weak, there are very few MMs accumulated at the stellar center following gravitational collapse. However, stars and planets can capture MMs from interstellar space during their life time after their birth. The number of the MMs captured is directly proportional to the surface area and the age of the stars and planets. Making use of the number of MMs captured from space (for stars and planets) and using the RC effect as the energy source, we will mow consider a series of juggling astrophysical phenomena including supernova explosion mechanisms of curious types and $\gamma$-ray burst mechanisms.

\section{Supernovae Explosion Mechanism driven by MMs}

The masses for the progenitors SNII, SNIB, SNIC, SLSN are respectively given by $(8-25) M_{\odot},(30-$ $60) M_{\odot},(80-150) M_{\odot}$, and $(200-1000) M_{\odot}$.

4.1 The number of MMs captured by massive stars during their life time

The time scale of the stars in the main sequence stage is about $90 \%$ of the life time for the stars. Due to hydrogen burning in the main sequence stage of the stars the stars is relatively stable and the stellar radius changes little during the main sequence stage. However, the stellar radius during the red giant stage of the stars will increases up to more than (10-100) times of the radius of the main sequence stars at least, although the time scale of the stars in the red giant stage is only about $10 \%$ of the life time for the stars. Thus, the total number of MMs captured from space by the progenitor of supernova in its life time is mainly in its red giant stage.

Making use of the radius and life time of the various types of supernovae progenitors during their main sequence stage, we may calculate the number of MMs captured during the main sequence life time and multiplied. By multiplying the factor 10, we may estimate the number of MMs captured by supernovae progenitors during their life time (actually this is a lower limit). The MMs accumulated at the central core of the various types of the supernovae are originate from the from MMs captured in flight by their progenitors, so their number is directly proportional to both the surface area and the life time of the progenitors mainly during their red giant stage. In the following we list the lower limit for the number of MMs contained in the deep interior of the core for the various types of supernovae (with typical masses)

$N_{m}=4 \pi R^{2} \phi_{m} t \approx 1.0 \times 10^{31}\left(\frac{\phi_{m}^{0}}{10^{-2} \phi_{m}^{\mathrm{up}}}\right)\left(\frac{R_{\mathrm{RG}}}{10^{3} R_{\odot}}\right)^{2}\left(\frac{t_{\mathrm{RG}}}{10^{6} \mathrm{Yr}}\right)$

where $R_{\mathrm{RG}}$ denotes the radius of the star in its red giant stage and $t_{\mathrm{RG}}$ is the life time during the red giant stage of the progenitor of supernova.

Because the MMs accumulated at the central core of the various types of the supernovae are originate from the from MMs captured in flight by their progenitorsso their number is directly proportional to both the surface area and the life time of the progenitors during their main sequence stage. These super-heavy MMs must fall and accumulated at the deep interiors of the central core. In the central core the number of the MMs reach the Newtonian saturation value such that $n_{m}(r) \approx \xi_{s} n_{\mathrm{B}}(r), \xi_{s} \approx 1.9 \times 10^{-32}\left(m_{m} /\left(10^{9} m_{p}\right)\right)$. In this way, may estimate the radius of the central core where the MMs accumulated as follows

$N_{m} \approx \frac{4 \pi}{3} r_{c}^{3} \zeta_{s} \overline{n_{\mathrm{B}}} \approx 1.3 \times 10^{24}\left(\frac{\phi_{m}^{0}}{10^{-2} \phi_{m}^{\mathrm{up}}}\right)\left(\frac{\overline{n_{\mathrm{B}}^{c}}}{n_{n u c}}\right)\left(\frac{r_{c}}{10^{6} \mathrm{~cm}}\right)^{3}$

where

$\left(\frac{r_{c}}{10^{6} \mathrm{~cm}}\right) \approx\left(\frac{\overline{n_{\mathrm{B}}^{c}}}{n_{n u c}}\right)^{-1 / 3}\left(\frac{N_{m}}{1.3 \times 10^{24}}\right)^{1 / 3}$

It is well known that the central density of massive stars is very low before supernovae explosion. At least during the hydrogen burning main sequence stage, from relations for the same mode of stellar structure, more massive stars have lower central densities. This kind of stellar structure will not change qualitatively during the subsequent nuclear burning stages. The central density of the Sun is $(50-100) \mathrm{g} / \mathrm{cm}^{3}$. From this it may be estimated that the central density of massive stars are about $10 \mathrm{~g} / \mathrm{cm}^{3}$ during their main sequence stage. In this way from Equ.(15), the radius of the central core where the MMs accumulated may be estimated be $r_{c} \approx\left(10^{5}-10^{7}\right) \mathrm{km}$, very much smaller than the radius of the massive stars of the progenitor of the SN. During the core collapse of the supernova, the MMs in the region within $\mathrm{r}$ collapse together with the hot plasma toward the center of the star by the strong electromagnetic interaction of the MMs with the plasma. However, during the gravitational collapse of the stellar deep central core the MMs accumulated within the radius $r_{c} \approx\left(10-10^{3}\right) \mathrm{km}$ may interact strongly with the 
high temperature plasma through the electromagnetic interaction

The MMs in the region within $\mathrm{r}$ collapse together with the hot plasma toward the center of the star. The condition for saturation $n_{m}(r) \approx \xi n_{\mathrm{B}}(r)$ is still maintained during the process of gravitational collapse. When the central density reaches nuclear density during the collapse. We may use Equ.(10, 11) to estimate the radius of the region for the accumulation MMs to be only $r_{c} \approx\left(10-10^{3}\right) \mathrm{km}$. In the absence of the RC effect the traditional theory for supernovae explosion is as follows. When core collapse of massive stars reaches nuclear densitythe non-relativistic degenerate nucleon gas dominates the core pressure (which is much larger than the relativistic and degenerate election pressure), matters no longer continue to collapse inwards and a strong outward rebound shock is formed. However, as we discussed in Section 1.1, traditional theory cannot trigger supernova explosion. On the other handin the presence of the RC effect, when the central density approaches the nuclear density, only a very small amount of the MMs (less than $10^{7}$ mole) accumulated in the central core of radius $r_{c} \approx\left(10-10^{3}\right) \mathrm{km}$ can trigger continuous nucleon decay. And the resulting RC luminosity with the increasing central density of the nucleons following the continue collapsing is drastically increased to exceed the Eddington luminosity. The corresponding radiation pressure far exceeds the non-relativistic nucleon degenerate pressure. The strong radiation pressure will make the entire star to expand outwards. In other words, the huge radiation pressure due to the $\mathrm{RC}$ effect can trigger supernovae explosion.

\subsection{The RC luminosity of various types of supernovae}

Using nucleon decay induced by MMs as the energy source, we will now discuss the explosion mechanisms of the various types of supernovae (SNII, SNIb, SNIc and and SLSN) and $\gamma$-ray bursts (including long ray burst and short $\gamma$-ray burst). As before, we adopt the parameter value $\xi \approx 50$. At the end of thermal nuclear evolution of the super-massive stars (SNIc and the progenitor of SLSN) the process of electron- positron pairs annihilation leads to the unstable collapse of the central core of such stars. When the baryon number density exceeds the nucleon density the MMs accumulated in the deep interior of the stellar core quickly trigger nucleon decay via the $\mathrm{RC}$ effect and the energy released is very high with highest efficiency. We now substitute the number of MMs accumulated at the central core of the various types of supernovae from Equs. (8) into Equ.(12) to obtain

$L_{m} \approx 2.5 \times 10^{43} a\left(\frac{\xi}{10^{2}}\right)\left(\frac{n_{\mathrm{B}}^{c}}{n_{n u c}}\right)\left(\frac{T_{c}}{10^{11} \mathrm{~K}}\right)^{1 / 2} \mathrm{ergs} / \mathrm{s}$ where

$a=\left(R_{\mathrm{RG}} /\left(10^{3} R_{\odot}\right)\right)^{2}\left(t_{\mathrm{RG}} /\left(10^{6} \mathrm{Yr}\right)\right.$

$\xi \equiv \sigma /\left(10^{-30} \mathrm{~cm}^{3}\right)\left(\phi_{\mathrm{m}}^{0} /\left(10^{-2} \phi_{\mathrm{m}}^{\mathrm{up}}\right)\right)$.

In Equ.(16) of the RC luminosity of supernova, parameter a is determined by both the radius, $R_{\mathrm{RG}}$, and the life time, $t_{\mathrm{RG}}$, of the progenitor of the $\mathrm{SN}$ in the red giant stage. The parameter $\xi$ is the product of five uncertain physical quantities. Although the two factors, $\sigma /\left(10^{-30} \mathrm{~cm}^{3}\right),\left(\phi_{\mathrm{m}}^{0} /\left(10^{-2} \phi_{\mathrm{m}}^{\mathrm{up}}\right)\right)$ are uncertain, but the typical value of the parameter $\xi$ in Equ.(16) may be taken as $\xi=100$ by comparing the heat flux for hot molten interior of the earth with the same Equ.(16) in the unified model ( see Section 5.2).

4.3 Estimate of the radius, main sequence life time and the parameter $a$ for the progenitor of SNII

If radiation pressure is neglected, the upper half main sequence masses are larger and the mass-radius relation is approximately $R \propto M^{2 / 3}$. The corresponding massluminosity relation for the upper half main sequence stars with larger masses are $L / L_{\odot} \approx\left(M / M_{\odot}\right)^{3.5}$. The continuous hydrogen burning $\left(4^{1} \mathrm{H} \rightarrow{ }^{4} \mathrm{He}\right)$ in the central region releases $26.73 \mathrm{MeV}$ with efficiency $7.1 \times 10^{-3}$. The hydrogen abundance of nascent stars is denoted by $X_{0}$ (where $X_{0}$ is roughly about 0.68 for the sun). When a fraction, $\mathrm{f}(f \approx 0.12)$, of the hydrogen of the entire star has converted into helium, the star leaves the main sequence. About $90 \%$ of the life time of stars are in the main sequence. The main sequence life time $t$ of a massive star with initial main sequence mass $M$ may be roughly written as

$t_{\mathrm{MS}}=\frac{7.1 \times 10^{-3} f M c^{2}}{L} \approx 1.1 \times 10^{10}\left(\frac{f X_{0}}{0.1}\right)\left(\frac{M}{M_{\odot}}\right)^{-2.5} \mathrm{Yr}$

The mass-radius relation and the mass-luminosity relation given above can be approximately applied to the progenitor of SNII(Their initial mass is less than $\left.30 M_{\odot}\right)$. When the initial main-sequence mass of a star is greater than $20 M_{\odot}$, the fraction of the radiation pressure to the total pressure begins to exceed $(25 \%-30 \%)$ and radiation pressure cannot be neglected .The radiation pressure increase very fast as the stellar mass increase. The effect of the radiation pressure on the stellar radius cannot be neglected. The stellar radius increase very fast as the stellar mass increases and the stellar radius clearly grows to exceed the previously mentioned limit for which the radiation 
pressure is neglected $\left(R \propto M^{2 / 3}\right)$. For the stars with still larger mass $\left(M>M_{\odot}\right)$ (They are the progenitors of SNIb SNIC SLSN), the radiation pressure dominates the total pressure and the stellar radius increases very fast as the stellar mass increases. For a supermassive star $\left(M>100 M_{\odot}\right)$, radiation pressure dominates with an approximate mass-luminosity relation $L \propto M$ (Shapiro et al. 1983). For these massive stars, the radiation pressure increases very fast as the masses are increased. Stellar winds are drastically strengthen and no clear mass luminosity relation exists. The stellar radius increases rapidly with increasing mass. We take the following crude approximation (hypothesis)

$$
\frac{R_{\mathrm{MS}}}{R_{\odot}}=10\left(\frac{M}{20 M_{\odot}}\right)^{\beta} \quad(\beta \approx 1.0-1.5),
$$

and we have

$$
\frac{R_{\mathrm{RG}}}{R_{\odot}}=10^{3}\left(\frac{M}{20 M_{\odot}}\right)^{\beta} \quad(\beta \approx 1.0-1.5),
$$

which for the radius of the progenitor of the $\mathrm{SN}$ with the red giant stage.

Main sequence life time for such super-massive stars can only be roughly estimated

$t_{\mathrm{MS}}=10^{7}\left(\frac{M}{20 M_{\odot}}\right)^{-1} \mathrm{Yr}$.

The life time of the red giant stage of such supermassive stars may be estimated as

$t_{\mathrm{RG}}=10^{6}\left(\frac{M}{20 M_{\odot}}\right)^{-1} \mathrm{Yr}$.

From Equ. (17), with the two Equs. (21, 23), we may crudely estimate the parameter $a$ by a relationship with the mass of the supermassive stars, $a=\left(M / 20 M_{\odot}\right)^{2 \beta-1}$. Substituting it into (16), the RC luminosity catalyzed by the MMs accumulated at the central core of the supernovae is obtained as following

$$
\begin{aligned}
L_{m} \approx & 2.5 \times 10^{43}\left(\frac{M}{20 M_{\odot}}\right)^{2 \beta-1}\left(\frac{\xi}{100}\right) \\
& \times\left(\frac{n_{\mathrm{B}}^{c}}{n_{\text {nuc }}}\right)\left(\frac{T_{c}}{10^{11} \mathrm{~K}}\right)^{1 / 2} \mathrm{ergs} / \mathrm{s}
\end{aligned}
$$

The core collapsing process is close to the free collapsing process (the inward collapsing velocity is about half of the free fall velocity). For more massive stars, the free collapsing velocity is faster and the more tightly compressed core, then leads to larger $n_{\mathrm{B}}^{c} / n_{n u c}$. Using the relation between the radius and mass of the degenerate compact stars $R \propto M^{-1 / 3}$, we speculate that the center density of collapsed core of the supernova is approximately $n_{\mathrm{B}}^{c} / n_{n u c} \propto\left(M / 20 M_{\odot}\right)^{2}$. Substituting it in to the Eq.(24), we obtain

$$
L_{m}^{\text {peak }} \approx 2.5 \times 10^{43}\left(\frac{M}{20 M_{\odot}}\right)^{2 \beta+1}\left(\frac{\xi}{100}\right)\left(\frac{T_{c}}{10^{11} \mathrm{~K}}\right)^{1 / 2} \mathrm{ergs} / \mathrm{s}
$$

Thus we have

$$
\begin{aligned}
& L_{m}^{\text {peak }} \propto\left(\frac{M}{20 M_{\odot}}\right)^{3} \quad(\beta=1) \\
& L_{m}^{\text {peak }} \propto\left(\frac{M}{20 M_{\odot}}\right)^{4} \quad(\beta=1.5)
\end{aligned}
$$

For the brightest known super luminous supernova (SLSN) (Dong et al. 2016), if the mass of the initial main sequence star for the progenitor of the SLSN exceeds $\left(10^{3}-10^{4}\right) M_{\odot}$ or more, then its radius during the main sequence stage is about $\left(10^{4}-10^{5}\right) R_{\odot}$, but its main sequence lifetime is very short, only $\left(10^{4}-10^{5}\right)$ years. Thus the parameter defined by (16) may reach $\left(10^{6}-10^{7}\right)$. The RC luminosity of the energy release rate will reach more than $\left(10^{49}-10^{50}\right) \mathrm{ergs} / \mathrm{s}$ when the core center density of the supernova exceeds the nuclear density. This explains the observed luminosity for the brightest super luminous supernova so far.

When the central density of the collapsed core exceeds the nuclear density, then the rates of energy release from the central core due to the RC effect for supernovas of types SNII, SNIb, SNIc and the brightest supernovae SLSN can all reach $10^{43} \mathrm{ergs} / \mathrm{s}$ or more. The huge radiation pressure drives the central core of the stars to violently expand outward to form SN, SNIb , SNIc, and SLSN. This is the effective mechanism for supernovae explosion driven by the MMs. With increasing mass of the progenitor for the series SNII, SNIb, SNIc and SLSN, the peak luminosity of the supernova rapidly increases with $\left(M / 20 M_{\odot}\right)^{(2 \beta+1)}$. The central density of the collapsed core of the supernova is approximately proportional to the square of the mass of the progenitor of the supernova.

4.4 The concrete mechanism for supernovae explosion

In order to explode the entire star violently, the RC luminosity must much exceed the Eddington's luminosity. Therefore, we have

$L \gg L_{E d d}=\frac{4 \pi c G M}{\kappa} \approx 1.3 \times 10^{38}\left(\frac{\kappa}{0.4}\right)^{-1}\left(\frac{M}{M_{\odot}}\right) \mathrm{ergs} / \mathrm{s}$

where $\kappa$ denotes opacity. At high temperatures Thomson scattering dominates $\kappa \approx 0.4 \mathrm{gcm}^{2}$. When conditionthe inequality (28) is satisfied, the corresponding 
radiation pressure is so huge that the stellar mantle, outer layer and the stellar atmosphere of the entire star are all violently ejected outwards. The highest speed of the drastic projection may reach $(1-2) \times 10^{4} \mathrm{~km} / \mathrm{s}$ - This is the observed scenario of supernovae explosion. As for how large the ratio of $L_{m} / L_{\text {edd }}$ must be in order to achieve supernovae explosion, detailed numerical simulation is really needed. Here we may roughly estimate the ratio

$b=L_{m}^{\mathrm{peak}} / L_{\mathrm{Edd}} \approx 2 \times 10^{4}\left(\frac{M}{20 M_{\odot}}\right)^{2 \beta-1}\left(\frac{n_{B}^{c}}{n_{\text {nuc }}}\right)$,

Therefore, the value of $\mathrm{b}$ has already reached 400 not yet reach $1 \%$ of the nuclear density even for the initial main sequence mass with $20 M_{\odot}$. Thus the RC luminosity of the supernova must be much greater than the Eddington's luminosity i.e. the condition Equ.(28) is satisfiedthe MMs accumulated at the central core of massive stars can continuously induce nucleon decay leading to huge RC luminosity and drive violent supernovae explosion.

We note that the Eddington luminosity increases with the masses of the supernovae progenitors of the series SNII, SNIb, SNIc, SLSN increase. In order to achieve supernovae explosionthe supernovae core must he highly compressed to exceed nuclear density such that the required RC luminosity is reached. Actually, during the gravitational collapse of massive stars election capture in iron at high density is very fast, both the resulting number of free elections and the degenerate election pressure drop very quick and the core collapse is close to free collapse (the inward collapsing speed in half that of the free collapse).

More massive stars have larger free collapsing speed, higher density of the stellar core, larger $n_{B} / n_{\text {nuc }}$ ratio and greater RC luminosity. Consequently, as the masses of the progenitors of the series SNII, SNIb, SNIc, SLSN increase, the peak value of the supernovae explosion becomes higher. The is basically consistent with general consensus.

In the following, we will discuss in more detail how the huge $\mathrm{RC}$ radiation pressure blow off the supernovae. In the region $r \leq r_{c}$, the RC luminosity may be written as

$$
\begin{gathered}
L_{m}(r)=\int_{0}^{r} 4 \pi r^{2} n_{m}(r) n_{\mathrm{B}}(r)\langle\sigma v\rangle m_{\mathrm{B}} c^{2} d r \\
=\int_{0}^{r} 4 \pi r^{2} \zeta_{s} n_{\mathrm{B}}^{2}(r)\langle\sigma v\rangle m_{\mathrm{B}} c^{2} d r \\
\approx 0.7 \times 10^{40}\left(\frac{\frac{n_{\mathrm{B}}^{c}}{n_{\text {nuc }}}}{L_{m}}\right)^{2}\left(\frac{r}{10^{6} \mathrm{~cm}}\right)^{3}\left(\frac{\sigma}{10^{-30} \mathrm{~cm}^{2}}\right)\left(\frac{T}{10^{11} \mathrm{~K}}\right)^{1 / 2}
\end{gathered}
$$

where $\overline{n_{\mathrm{B}}^{c}}$ denotes the average nucleon number density of the central core in which the MMs are accumulated. The temperature of the central core is $10^{11} \mathrm{~K}$ with corresponding nucleon thermal velocity $5 \times 10^{9} \mathrm{~cm} / \mathrm{s}$. Since all the MMs are concentrated within $r \leq r_{c}$, there are no magnetic monopoles outside $r_{c}$, therefore the RC luminosity for $r>r_{c}$ is constant, namely

$$
\begin{aligned}
& L_{m}(r) \approx 0.7 \times 10^{40}\left(\frac{\overline{n_{\mathrm{B}}^{c}}}{n_{n u c}}\right)^{2}\left(\frac{r_{c}}{10^{6} \mathrm{~cm}}\right)^{3} \\
& \times\left(\frac{\sigma}{10^{30} \mathrm{~cm}^{2}}\right)\left(\frac{T}{10^{11} \mathrm{~K}}\right)^{1 / 2} \quad\left(r>r_{c}\right)
\end{aligned}
$$

The huge radiation pressure generated by the $\mathrm{RC}$ luminosity may be determined by

$$
-\frac{d P_{r}(r)}{d r}=\frac{\kappa \rho(r) L_{m}(r)}{4 \pi r^{2} c},
$$

Substituting (31) and (32) into (33), we obtain

$$
\begin{aligned}
&-\frac{d P_{r}(r)}{d r}= 2.0 \times 10^{42}\left(\frac{\xi}{100}\right)\left(\frac{\kappa}{0.4}\right)\left(\frac{\overline{n_{\mathrm{B}}^{c}}}{n_{n u c}}\right)^{3}\left(\frac{r_{c}}{10^{6} \mathrm{~cm}}\right)\left(\frac{r}{r_{c}}\right), \\
&-\frac{d P_{r}(r)}{d r}= 2.0 \times 10^{45}\left(\frac{\xi}{100}\right)\left(\frac{\kappa}{0.4}\right)\left(\frac{n_{\mathrm{B}}^{c}}{n_{n u c}}\right) \\
& \times\left(\frac{\overline{n_{\mathrm{B}}^{c}}}{n_{\mathrm{nuc}}}\right)^{2}\left(\frac{r_{c}}{10^{6} \mathrm{~cm}}\right)^{2}\left(\frac{r}{r_{c}}\right)^{-2},
\end{aligned}
$$

where the conditions for Equs. $(34,35)$ are corresponding to $r \leq r_{c}$ and $r>r_{c}$.

In the collapsing central region the gas pressure is dominated by the non-relativistic neutron degenerate pressure with equation of state

$$
P_{g}(r) \approx K \rho^{5 / 3} \quad\left(K \approx 5.4 \times 10^{9} \quad \text { c.g.s }\right),
$$

and the gas pressure gradient is

$$
-\left(\frac{d P_{g}(r)}{d r}\right) \approx 1.1 \times 10^{34}\left(\frac{\rho(r)}{\rho_{\mathrm{nuc}}}\right)^{2 / 3}\left(-\frac{d \rho(r) / d \rho_{\mathrm{nuc}}}{d r}\right),
$$

In stellar interior even if we take the high density gradient $d \rho(r) / d \rho_{\text {nuc }} \approx 10^{5} \mathrm{~km}$ (a fast decrease of nuclear density to $10^{8} \mathrm{~g} / \mathrm{cm}^{3}$ in a distance of $10 \mathrm{~km}$ ) the radiation pressure generated by the $\mathrm{RC}$ effect in the supernovae core $<10^{3} \mathrm{~km}$ is far larger than the nonrelativistic neutron degenerate pressure. This means that we can neglect the non-relativistic neutron degenerate pressure in our discussion, i.e. the total pressure of stellar interior $P(r) \approx P_{r}(r)$. According to the theory and dynamic equation of stars, we have

$\rho\left(\frac{d^{2} r}{d t^{2}}\right)=-\left(\frac{d P_{r}}{d r}\right)-\left(\frac{G M(r)}{r^{2}}\right) \rho(r)$, 
when

$-\left(\frac{d P_{r}}{d r}\right) \gg\left(\frac{G M(r)}{r^{2}}\right) \rho(r)$,

The huge radiation pressure will make the stellar matter within $\mathrm{r}$ to violently ejected outwards. We will introduce the parameter $b^{\prime}$ to depict the necessary condition that the radiation pressure is sufficient to trigger supernovae explosion

$-\left(\frac{d P_{r}}{d r}\right) \geq b^{\prime}\left(\frac{G M(r)}{r^{2}}\right) \rho(r) \quad\left(b^{\prime} \approx 10-10^{2}\right)$,

where we assume that $b^{\prime}$ in Equ.(40) is roughly equal to the value b in Equ.(29)).

When the density of supernovae core satisfied condition(the inequality (29), the inequality (39) may also be satisfied and the series of supernovae SNII, SNIb, SNIc, and SLSN explosions can succeed. Our important conclusions are as follows, the necessary and sufficient condition for the magnetic monopoles induced nucleon decay and the resulting $\mathrm{RC}$ luminosity and radiation pressure sufficiently strong to violently eject the stellar matter is that the density of the collapsing core must reach near the nuclear density and above.

The density of the collapsing core increases as the initial main sequence mass of the supernovae progenitor series SNII, SNIb, SNIc, and SLSN increase. From Equ.(16) and Equ. (25) we note also that the RC luminosity and the peak luminosity increase very fast as the initial main sequence mass increases.

Our model is different from the standard model SNII for supernovae explosion. In Section 1, we already mentioned the reasons for the unsuccessful SNII model. Firstly, the instantaneous explosion mechanism for supernovae explosion fails due to the energy of the outgoing rebound shock being exhausted before the matters in the outer layers are entirely and completely destroyed by the $\gamma$-ray photons (from the rebound shock). In our theory, the MMs accumulated in the supernovae inner core continuously trigger nucleon decay with huge energy release. When the density of the collapsing core reaches or above nuclear density, the $\mathrm{RC}$ luminosity may reach $10^{41}-10^{42} \mathrm{ergs} / \mathrm{s}$ or above. This luminosity is generated continuously and it provides powerful radiation flux until supernovae explosion is achieved. This RC luminosity can only be weaken after supernovae explosion when the SNII theory. The energy of the outgoing shock is completely exhausted before the outer iron core breaks up. Clearly, similar problem cannot happen in our theory.

Secondly, our approach is also different from the neutrino delayed explosion mechanism proposed by Wilson et al. (1988) . supernovae explosion cannot be achieved because the interaction between the neutrino flux and matter is too weak to break the outer atmosphere and supernovae explosion (Buras et al. 2003; Liebendoerfer et al. 1992). In our theory the successful supernovae explosion is achieved through the continuous generation of the RC luminosity and the huge radiation pressure. MMs triggered nucleon decay play the key role. Of course, in our explosion scenario, the transformation of the gravitational potential energy of $\left(10^{52}-10^{53}\right)$ ergs convert into the thermal energy. The powerful neutrino flux of energy $10^{52}$ ergs and above generated by the conversion of $(u, d)$ and $(\mathrm{u}, \mathrm{d}, \mathrm{s})$ quarks in the high temperature environment of the nascent neutron star are the same as that of the standard SNII model(Dai et al. 1995).

\subsection{Weak explosion or dark explosion of supernova}

If the RC luminosity is not much higher than the Eddington's luminosity of the star during the collapse of the central core, then the resulting explosion is rather weak and may be referred to as dark explosion. The supernovae remnant Cas A (it is only $3.4 \mathrm{Kpc}$ away from the Earth), for instance, corresponds supernovae with maximum visual luminosity of $5^{m}$, which exploded in 1680. The remnant neutron star of this explosion was discovered in 1999. It is very likely that this is a concrete example of weak explosion.

By researches via the observation of the Chandra Xray satellite, recently, the interstellar nebula G1.9+0.3 is considered as a supernova remnant exploded about 110 years age, but it has never been reported. It may be the dark explosion. These dark supernova may be formed from the direct collapse of the white dwarfs. Their density is not very high during supernova explosion, so the RC luminosity is only slightly greater than Eddington luminosity and they are weak explosion.

More recently, NASA reported on 28th May, 2017 by blasting news in the network. A star N6946-BH1 suddenly disappears from astronomical observations in 2015 (It also totally disappears even though from observations by both Hubble Space Telescope and Spitzer Space Telescope), although it is rather luminous in 2007 and its brightness begun to strengthen in 2009. People guess that it has directly collapsed to a black hole according to the popular idea. But it is an typical example for the weak explosion in our model.

\subsection{The remnant neutron stars after supernovae explosion}

MMs induce nucleon decay in the supernovae core with huge energy release. The resulting super-strong radiation pressure resists the gravitation collapse of the supernovae core and the central core cannot continue to 
collapse. The density of the core cannot approach infinity. Instead, the matters in the supernovae core are driven outwards by the huge radiation pressure and the density of the supernovae core drops quickly.

When the RC luminosity is strong the velocity of the large amounts of matter projected outwards exceeds the escape velocity. Thus, during supernovae explosion, the mantle, outer layer and the outer atmosphere are all ejected far away from the star. But, when the RC luminosity and the corresponding radiation pressure are lowered, the matters in the stellar interior with ejection velocity less than the escape velocity and especially the matters in the deep supernovae core begin to fall towards the center. But the resulting gravitational collapse will not lead to infinite density to form black hole. This is because there are always a few MMs still remain in the deep interiors of the supernovae core even though large amounts of the MMs are quickly ejected outward outside the star. The remaining MMs interact strongly with the high temperature plasma in the supernovae core through electromagnetic interaction. These remnant super heavy MMs in the innermost supernovae core can still continuously to trigger nucleon decay to generate the RC luminosity far lower than the Eddington luminosity of the remnant star. Finally, according to Eq.(28), some kind of stable dynamical equilibrium may be reached

$$
\begin{aligned}
L_{E d d}= & 1.3 \times 10^{38}\left(\frac{\kappa}{0.4}\right)^{-1}\left(\frac{M}{M_{\odot}}\right) \\
& \gg L_{m}=\frac{4 \pi}{3} r_{c}^{3} \zeta_{s}\left(n_{\mathrm{B}}^{c}\right)^{2}\langle\sigma v\rangle m_{\mathrm{B}} c^{2},
\end{aligned}
$$

We may use Eq.(14) and Eq.(41) to estimate of the central core

$\frac{n_{\mathrm{B}}^{c}}{n_{\text {nuc }}} \ll 10^{-2} \eta\left(\frac{M}{M_{\odot}}\right)$,

where

$\eta=\left[\left(\frac{m_{m}}{10^{9} m_{p}}\right)\left(\frac{\sigma}{10^{-30} \mathrm{~cm}^{2}}\right)\left(\frac{N_{m}}{10^{24}}\right)\right]^{-1}$,

Only a few magnetic monopoles remains in the core of the nascent neutron star $\left(N_{m}\right.$ is much less than $10^{24}$ ) because most of the magnetic monopoles in the collapsed core of the supernova are thrown out with the plasma by the strong electro-magnetic interaction. From this we can estimate the average matter density in the innermost core at dynamical equilibrium to be only $\rho_{c} \ll 10^{-2} \rho_{\text {nuc }}$. Outside the innermost core with radius $r_{c}$, the mass of the matter contained increases very fast and $L_{\mathrm{Edd}}$ also drastically increase. But the RC luminosity $L_{m}$ original from the innermost core can no longer increase, so $L_{m} \ll L_{e d d}$, matter in the stellar interior can no longer be driven outwards. Actually, RC luminosity $L_{m}$ decreases very fast as the density of the supernovae core drastically decreases. From Eq.(35), we note that the radiation pressure decreases very fast as the ratio $\overline{n_{\mathrm{B}}^{c}} / n_{\text {nuc }}$ decreases. It is seen from Eq. (42) that if $\overline{n_{\mathrm{B}}^{c}} / n_{\text {nuc }} \ll 10^{-2}$, the corresponding RC luminosity greatly decreases such that the matter outside the innermost core containing MMs (with radius $r_{c}$ ) can no longer be driven out. At this time the strong gravitation due to the matter in the thick outer layer can compress the matter inside the star to nuclear density to form neutron stars or super-massive neutron stars. Using MMs induce nucleon decay as the energy source, neutron stars cannot collapse to form black holes no matter how massive they are.

The remnants after supernovae explosion are stellar objects similar to neutron stars. The density in the innermost core is not high. Because the remnant MMs can continuously induce nucleon decay to provide energy source and huge radiation pressure so that this stellar object cannot collapse to form a black hole. Other important prediction from our analysis is that there is no upper limit for the mass of the neutron stars. At least, there is no generally accepted upper limit of $25 M_{\odot}$. A massive neutron star origins from the supernova with a super massive progenitor. It is well known that the probability of the birth of super massive stars is very small according to the initial mass distribution function of Salpeter. Up to now, the number of neutron stars that their masses have been measured or (have been estimated) in close binaries is less than 20. The above result without the upper limit of $25 M_{\odot}$ is not inconsistence with astronomical observations.

We would like to emphasize that the only difference between the neutron stars formed from the standard SNII model and our model is the innermost structure with MMs that we proposed. As for SNIb, SNIC, and SLSN with their much more massive progenitors would not collapse to form black holes with infinite density. Instead, they form more massive neutron stars with remnant MMs accumulated in the deep interior of the supernovae core. Since the long $\gamma$-ray burst originated from supernovae SNIc, so that our model of supernovae explosion driven by MMs also apply. 
5 The other related puzzle of our model of supernova driven by magnetic monopoles

5.1 The problem of the heat source of the core molten state in the Earth interior

\subsubsection{The puzzle of a hot molten state for the core of the Earth}

It is well known from the eruption of the volcano and the hot magma that the core of the earth is in a hot molten state, The core of the Earth is very hot, but the shell temperature is relatively lower. For instance, the temperature in the surface of the earth is $300 \mathrm{~K}$. This state of molten core can't be caused by absorbing energy from the solar radiation. Thus, it must contain another energy sources in the interior of the earth. A popular idea on this question is that the huge thermal energy in the Earth's core origins from collapsed core in the formation process (for an example, see the book from Wikimedia). However, you may see that this idea is questionable by following discussion.

The total thermal energy from the interior of the Earth is given by

$E_{k} \approx \frac{4 \pi}{3} R_{c}^{3} \overline{\rho_{c}} N_{A} k \bar{T} / \bar{\mu}$,

where the core radius of the earth is $R_{c} \approx 2 \times 10^{3} \mathrm{Km}$. The core density of the earth is $\overline{\rho_{c}} \approx 13 \mathrm{~g} / \mathrm{cm}^{3}$. The core temperature of the earth is $\overline{T_{c}} \approx 6 \times 10^{3} \mathrm{~K}$. The average molecular weight in the core of the Earth is $\bar{\mu} \approx 30$. Thus, we have $E_{k} \approx 0.7 \times 10^{37}$ ergs.

The total heat flow from earth's interior to surface crust is about $47 \mathrm{TW}$ (i.e. $J_{r} \approx 4.7 \times 10^{20} \mathrm{ergs} / \mathrm{s}$ ) (Davies et al. 2010). The time scale of outward transport thermal energy, in a case without energy source, from the interior of the earth is given by

$t_{t h}=\frac{E_{T}}{J_{r}} \approx 1.5 \times 10^{16} s \approx 0.5 \times 10^{9} \mathrm{Yr}$,

This time scale is much shorter than the age of the earth. That is, it is impossible that the huge thermal energy in the Earth's core is left over from the birth of the Earth. Therefore, we conclude that there must be some heat source in the earth core. We first discuss that if the radioactive elements in the interior of the earth may provide enough energy in the time scale $t_{t h}$. The heat energy is mainly produced from the three most important radioactive elements. The heat release rate by the three naturally radioactive elements are $0.78 \mathrm{cal}(\mathrm{yrg}(\mathrm{U}))^{-1}, 0.20 \mathrm{cal}(\mathrm{yrg}(\mathrm{Th}))^{-1}, 2.6 \times$ $10^{-6} \mathrm{cal}(\mathrm{yrg}(\mathrm{K}))^{-1}$ for the uranium series, the thorium series, and the actinium series, respectively (Allen et al.
1956). As an example, we may discuss the most important radioactive elements Uranium. According to some parameters of the abundance of atomic number $n(\mathrm{U}) / \mathrm{n}(\mathrm{H}) \approx 10^{-12}$, the mass abundance $m(\mathrm{U}) / \mathrm{m}(\mathrm{H}) \approx 10^{-10}$, and the mass of the earth $6 \times 10^{27} \mathrm{gm}$, we can know the total mass of radioactive elements Uranium are about $6 \times 10^{17} \mathrm{gm}$. Thus, the total heat release of all radioactive elements Uranium from the interior of the earth within $0.5 \times 10^{9} \mathrm{Yr}$, is given by $E_{\mathrm{U}} \approx 10^{34} \mathrm{ergs} \approx 1.5 \times 10^{-3} \mathrm{E}_{\mathrm{T}}$

Synthesizes the above analysis, our conclusion has two. One is that radioactive energy far cannot provide the source of energy of the earth core melt state. Another is that the thermonuclear reaction cannot be ignited due to the lower temperature of the Earth interior(i.e. $6 \times 10^{3} \mathrm{~K}$ ). Thus, it is necessary for us to find new energy source for the melt state of the Earth core.

\subsubsection{The puzzle of a hot molten state for the core of the Earth}

MMs accumulated in the central core of the earth and the RC luminosity generated by nucleon decay induced by the MMs may be computed from Eq.(9). The temperature of the earth at its center is about $T \approx 6 \times 10^{3} \mathrm{~K}$. According to our model for stellar objects containing MMs, the RC luminosity generated by the number of MMs that are captured by the Earth from interstellar space since its birth may be estimated from Equ.(16)

$$
\begin{gathered}
L_{m} \approx 3.0 \times 10^{18}\left(\frac{\sigma}{10^{-30} \mathrm{~cm}^{2}}\right)\left(\frac{\phi_{m}^{0}}{10^{-2} \phi_{m}^{\mathrm{pp}}}\right) \\
=3.0 \times 10^{18} \xi \mathrm{ergs} / \mathrm{s},
\end{gathered}
$$

where $\xi=\left(\frac{\sigma}{10^{-30} \mathrm{~cm}^{2}}\right)\left(\frac{\phi_{m}^{0}}{10^{-2} \phi_{m}^{\text {पp }}}\right)$, Compared with $J_{r}=$ $4.7 \times 10^{20} \mathrm{ergs} / \mathrm{s}$, if the outward heat flow from the Earth's interior is provided by the RC effect we must choose the parameter $\xi \approx 100$. In other works, starting from the actual date of the hot molten interior of the Earth, we are able to determine the parameter $\xi \approx 100$. In sections 4 , we will use this value of $\xi$ to determine the RC luminosity of supernova explosion.

By the way, we may estimate the number of MMs captured by Jupiter after its birth (the age of Jupiter is about 450 million years )

$N_{m} \approx 5.0 \times 10^{19}\left(\frac{\zeta_{m}^{0}}{\zeta_{s}}\right)\left(\frac{n_{\mathrm{B}}^{0}}{1 \mathrm{~cm}^{-3}}\right)\left(\frac{\nu_{m}}{10^{-4} c}\right)$

There are also the energy source problems for Jupiter, the corresponding energy production rate is $L_{m} \approx 10^{21} \mathrm{ergs} / \mathrm{s}$. Whether this is correct or not may be tested by future study. Whether this is well or not may be tested by future study. 
5.2 On Cooling of white dwarfs

\subsubsection{The puzzle of White Dwarf cooling}

The effective temperatures of most of the white dwarfs (hereafter WDs) are in the range of $5.5 \times 10^{3}-4 \times 10^{4} \mathrm{~K}$, only few WDs have effective temperatures outside this range. Their spectral types corresponding to these white dwarfs are from $\mathrm{O}$ to $\mathrm{K}$.

But individual ones is for M types (Zhu 2003). Why are the spectral types for most of WDs above types A (i.e. O, B, A) , but only are F, G, K for few WDs, and few of WDs have the spectral types of later M, and $\mathrm{N}$, whose surface temperature are less than $3 \times 10^{3} \mathrm{~K}$.

The temperature in the interiors of WDs is $10^{6} \mathrm{~K}$ with total thermal energy less than $10^{47}$ ergs. The radius of white dwarf star is about $10^{4} \mathrm{~km}$ with surface temperature $T \approx 5 \times 10^{3}-4 \times 10^{4} \mathrm{~K}$. Since most of WDs have surface temperature $1 \times 10^{4} \mathrm{~K}$, we therefore adept the typical surface temperatures of WDs as $1 \times 10^{4} \mathrm{~K}$. The radiation luminosity of WDs is roughly $L_{\mathrm{rad}} \approx 10^{31} \mathrm{ergs} / \mathrm{s}$, so the typical cooling time scale of WDs is about $10^{6} s \approx 3.3 \times 10^{8} \mathrm{Yr}$. In the other words, WDs. Should cool down within 400 million years, then why no late type $\mathrm{M}$ and type late $\mathrm{N}$ WDs with surface temperatures less than $3000 \mathrm{~K}$ ever been observed? What are the heat sources of these late type WDs? Astronomers did not discuss this problem. This is because no other physical process could provide such heat source. Adopting a model of celestial objects containing the MMs and taking the $\mathrm{RC}$ effect into account as an energy source(the MMs may catalyze nucleon decaying), we solve the explosion mechanism in this paper for all kinds of supernova including $\gamma$ ray burst and the energy sources in the core of the earth and White Dwarfs.

\subsubsection{The RC luminosity in White Dwrafs interiors}

The MMs captured by WDs (including progenitor stars) from the universe (accumulated at the stellar core) during their life time and using the $\mathrm{RC}$ effect as the energy source may naturally explain the existence of internal energy source in white dwarfs so that white dwarf cooling can be stopped. According to the standard theory of stellar evolution the WDs observed now originated from pre-main sequence stars with masses $(2-8)$. After hydrogen and helium burning stage these stars with low and middle mass lost their outer layer through the AGB star stage, then their central cores become white dwarfs.

We have shown in Section 3.3 that the total number of MMs captured from space by the progenitor of the white dwarfs is about 10 times of one calculated by Eq.(5) for this progenitor during its main sequence stage with a radius about $2 \mathrm{R}$ ? Thus from Eq.(5), we may estimate the total number of MMs captured from the space by the progenitor of white dwarfs during their life time to be

$N_{m} \approx 1.0 \times 10^{28}\left(\frac{\phi_{m}^{0}}{\phi_{m}^{\mathrm{up}}}\right)\left(\frac{R_{\mathrm{RG}}}{R_{\odot}}\right)^{2}\left(\frac{t_{\mathrm{RG}}}{10^{8} \mathrm{Yr}}\right)$

where $R_{\mathrm{RG}}$ is the radius of the progenitor of the write dwarf in the red giant stage. Its typical value is taken as $100 R_{\odot}$ ? and the time scale of their red giant stars with mass greater than $2 \mathrm{~m}$ ? is taken about $10^{7}$ years.

These captured super-massive MMs accumulated at the deep interior of the stellar core and the resulting $\mathrm{RC}$ luminosity is again given by Eq.(4). During the hydrogen burning main sequence stage, helium burning red giant stage and the AGB stage for their progenitors, of the white dwarfs, the stellar core density is respectively $\rho_{c}=10,10^{3}, 10^{6} \mathrm{~g} / \mathrm{cm}^{3}$ or $n_{\mathrm{B}}^{c}=$ $10^{25}, 10^{27}, 10^{30} \mathrm{~cm}^{3}$, and the central temperature is respectively $T_{c}=3 \times 10^{7}, 2 \times 10^{8}, 10^{8} \mathrm{~K}$, with corresponding thermal speed, $\overline{\nu_{T}} \approx 10^{8} \mathrm{~cm} / \mathrm{s}$ and luminosity $L_{m} \approx 5 \times 10^{26} \xi, 5 \times 10^{28} \xi, 5 \times 10^{31} \xi \mathrm{ergs} / \mathrm{s}$.

These luminosity is far lower than the corresponding luminosity provided by thermal nuclear reaction during stellar evolution (where $\xi \approx 50$ ). After the evolutionary stage of white dwarfs, however, thermal nuclear burning in the interior of white dwarfs $T_{c} \approx(1-3) \times 10^{6} \mathrm{~K}$ stopped and there are no more energy sources. The central temperature of white dwarfs with corresponding thermal velocity $\overline{\nu_{T}} \approx 10^{7} \mathrm{~cm} / \mathrm{s}$. The typical value of the central density for white dwarfs is $\rho_{c}=10^{7} \mathrm{~g} / \mathrm{cm}^{3}$ or $n_{\mathrm{B}}^{c}=10^{30} \mathrm{~cm}^{3}$. Then we obtain from Eq.(16)

$L_{m} \approx 1.2 \times 10^{33} \xi\left(\frac{R_{\mathrm{RG}}}{R_{\odot}}\right)^{2}\left(\frac{t_{\mathrm{RG}}}{10^{8} \mathrm{Yr}}\right)$

where $R_{\mathrm{RG}}$ is the radius of the progenitor of white dwarfs (i.e., the main sequence star) $t_{R G}$ is the life time of the main sequence star. If we use MMs induced nucleon decay as the energy source to reach the white dwarfs luminosity $L_{\mathrm{rad}} \approx 10^{31} \mathrm{ergs} / \mathrm{s}$, we are required to choose

$\xi\left(\frac{\nu_{m}}{10^{-4} c}\right)\left(\frac{R_{\mathrm{RG}}}{R_{\odot}}\right)^{2}\left(\frac{t_{\mathrm{RG}}}{10^{8} \mathrm{Yr}}\right) \approx 2$

If we also use the $\mathrm{RC}$ effect to provided the energy source for the molten hot core of the earth, we must choose $\xi \approx 50$ as we did before consequently

$\left(\frac{\nu_{m}}{10^{-4} c}\right)\left(\frac{R_{\mathrm{RG}}}{R_{\odot}}\right)^{2}\left(\frac{t_{\mathrm{RG}}}{10^{8} \mathrm{Yr}}\right) \approx 0.04$

This is a constraint on the radius and life time of the progenitor of white dwarfs (i.e., the constraint on the 
main sequence star). The white dwarfs luminosity are really in the range $10^{29}-10^{35} \mathrm{ergs} / \mathrm{s}$, If we also use the $\mathrm{RC}$ effect to provide the energy source for the molten hot core of the earth, we must choose $\xi=100$. Thus we may get a constraint on the radius and life time of the progenitor of white dwarfs during the red giant stage

$$
\left(\frac{R_{\mathrm{RG}}}{R_{\odot}}\right)^{2}\left(\frac{t_{\mathrm{RG}}}{10^{7} \mathrm{Yr}}\right) \approx\left(10^{-4}-10\right)
$$

Equ.(40) is a reasonable constraint. We note that the observed date for both molten state of the earth's core and the white dwarfs luminosity are accurate and reliable. Therefore the value of $\xi$ that we derived and the constraint condition (40) are very reasonable.

5.3 The problem for the possible association of gravitational waves and the short Gamma-ray burst

Recently, the study of the astrophysics of gravitational waves has attracted considerable attention. The international high frequency gravitational Wave Detector project LISA (for the detection of gravitational waves generated by the collision and merge of two massive AGNs with super-massive black holes) is now in active planning for space detection. The collision and merge of two smaller compact stellar objects (black hole, neutron star and white dwarf) may also produce gravitational waves. To this goal, the project for ground based observations called Advanced LIGO has already begin to accumulated large amounts of detected data LIGO already announced the detection of gravitational waves GW150914 in February 12, 2016 (Abbott et al. 1994). Using the model of the collision of two black holes with masses $36_{-4}^{+5} M_{\odot}$ and $29_{-4}^{+4} M_{\odot}$ respectively, the LIGO astronomers attempted to fit the formation of a final black hole with mass $62_{-4}^{+4} M_{\odot}$ after the generation of gravitational waves. The total energy released by the gravitational waves is approximately $3_{-0.5}^{+0.5} M_{\odot}$.

A few days later (i.e., February, 16, 2016), the $\gamma$-ray burst detecting group, Fermi GBM in the U.S published a paper (Connaughton et al. 2016) They reported that a weak and short $\gamma$-ray burst (lasting one second) was almost simultaneously detected ( 0.4 second later) in the direction of the gravitational wave. From the fact that the non-thermal luminosity is $10^{49} \mathrm{ergs} / \mathrm{s}$ in the wave zone $(1 \mathrm{KeV}-10 \mathrm{MeV})$, they asserted that this $\gamma$-ray burst is associated with the LIGO gravitational wave event GW150914.

But Lyutikov et al. (2016) suggested a different view point concerning the association of the gravitational wave event with the $\gamma$-ray burst event. First, the $\gamma$ ray burst associated with the gravitational wave event was not detected by another X-ray space detector INTERGRAL. (However, the sensitivity in the $50 \mathrm{KeV}$ energy range of the INTERGRAL X-ray space detector is not enough). The author made a detailed analysis about all the possible electromagnetic radiation mechanisms in the environment of a black hole with accreting plasma and concluded that the observed luminosity of $10^{49} \mathrm{ergs} / \mathrm{s}$ is several order of magnitudes higher than that can be provided by all the possible mechanisms under consideration. From this he concluded that the $\gamma$-ray burst event observed by Fermi GBM observations is not associated with the gravitational wave event GW150914. The author believes that these are two mutually independent coincident events in space and time.

Whether or not the gravitational wave event is associated with the $\gamma$-ray burst event? If the $\gamma$-ray burst event observed by Fermi GBM is real, then how to explain the association of the gravitational wave event with the $\gamma$-ray burst event? In his exotic theoretical model, Zhang quickly gives the answer (Zhang $2016 \mathrm{a}, \mathrm{b})$. If one of the two merging black holes carries enough and large amounts of electric charges (reaching $10^{-4}$ of the critical charge $\left.Q_{c}\right) Q \approx 10^{-4} Q_{c}$ and $Q=1.0 \times 10^{31}\left(M / 10 M_{\odot}\right)$ e.s.u, then not only gravitational waves can be generated but also triggering the production of short $\gamma$-ray burst and fast radio burst. Zhang (2016a,b) therefore concluded that the $\gamma$-ray burst event observed by the Fermi GBM observations is associated with the LIGO gravitational wave event GW150914.

Zhang also proposed that the gravitational wave event, the short $\gamma$-ray burst event and the fast radio burst event are somehow associated with each other (see Zhang $(2016 \mathrm{a}, \mathrm{b})$ ). The key problem is that the charged black hole must carry large amounts of charges. No body has never discussed how such highly charged black holes are formed. The highest saturation value for the charges carried by a Newtonian stellar object is roughly only about $5.2 \times 10^{12}\left(M / 10 M_{\odot}\right)$ e.s.u.

The Newtonian saturation value of electric charges for a charged celestial body is defined as follows. If a celestial body carries a positive charge and when the Coulomb electrostatic repulsive force acting on a proton is equal to the Newtonian gravity of the proton, the electric charge of the celestial body is called as the Newtonian charged saturation of protons. If the celestial body carries negative charges, the Newtonian charged saturation of electrons is about 1/1840 of one of protons for the celestial body with positive charge.

The critical charge of RN black hole is that when the electric quantity of the black hole reaches the value, the visual interface of the black hole disappears. If massive 
stellar objects really carry huge electric charges before they gravitationally collapsed to charged black holes, it may lead to unthinkable spectacular phenomena due to electrical polarization effects. It is difficult to believe that highly charged massive black holes really exist.

In our theoretical framework these two events are maturely associative. Short $\gamma$-ray bursts are generally believed to originate from the collision of neutron stars. The remnant neutron stars after supernovae explosion may also contain some magnetic monopoles in remnant neutron stars are evidently less than that in the progenitor stars. Free MMs can also be captured from space after the formation of neutron stars.

In our model of stellar objects containing MMs, there is a strong radial magnetic field. Hence, during the collision and merge of the two compact stellar objects (with same or apposite polarity) both gravitational and electromagnetic interaction can participate leading to radial attraction and repulsion. In the general frame work of the two different models (the standard black hole model and our model of super-massive stellar objects containing MMs) it is expected that the wave forms of the gravitational waves generated by the collision and merge of two AGNs (our model) or the collision and merge of two compact objects (black hole, neutron star, white dwarf) (the standard model) are rather different.

The collision and merge of two black holes can only generate gravitational waves. But no $\gamma$-ray burst can be simultaneously generated. However, the collision and merge of two compact and massive stellar objects containing MMs (with central density far exceed nuclear density reaching $\left(10^{3}-10^{5}\right) \rho_{n u c}$ or more the resulting RC luminosity may produce $10^{49} \mathrm{ergs} / \mathrm{s}$ and simultaneously generate short $\gamma$-ray burst and large number of charged particles via nucleon decay induced by the MMs. The radial magnetic field from the MMs may in turn generate electric field in a spinning stellar object to accelerate the charged particles. The radial magnetic field lines may be distorted. Radio waves may be generated when charged particles are ejected along curved magnetic field lines. It is possible that the production of fast radio burst (FRB) follows. From the above scenario, the short $\gamma$-ray burst occurred within a very short time interval after the appearance of the gravitational waves. If the two events observed are reliable, it seems advantageous to the validity of our model and lend support to the key roles played by the MMs.

Of course, similar to the weak explosion and the dark explosion of supernova, the short $\gamma$-ray burst may not be observed, if its RC luminosity is not much more than the Eddington's luminosity. The second weaker LIGO gravitational wave event GW170104 without short - ray burst may correspond to this situation in our model.
We will endeavor to further study and propose that if the association of $\gamma$-ray bursts with the production of gravitational waves is observed again, the association of the two events are real and it may be regarded as the observational evidence for the existence of MMs.

\section{Conclusions and outlooks}

The series of astrophysical phenomena discussed in our paper has been considered by astronomers and scientists as important but puzzling problems in the recent half century. Especially the explosion mechanisms for the various types of supernovae are the central topics to study in the recent half century and no convincing solutions to these puzzles has ever been found. Making use of the two ideas of the spatial flux of magnetic monopoles and nucleon decay induced by MMs (RC effect) as well as the theoretical formulae in our papers (Peng et al. 1985a, b) , we are able to explain the thermal energy source at the earth's central core, the heat source needed in white dwarf interiors and the explosion mechanisms for SNII, SNIb, SNIc, SLSN supernovae explosion and Gamma-ray bursts. We would like to emphasize that in our model we just take the parameter, $\xi$ (in Eq. (18)), as determined from the Earth's thermal flux.

It seems that our unified treatment of these puzzling issues has no doubt convinced us that the idea of MMs and the RC effect are reasonable and powerful tools for future investigation. We believe and we hope that our suggestions can attract considerable attention and leading to another round of interest to study MMs .

\section{Acknowledgements}

We would like to thank the anonymous referee for carefully reading the manuscript and providing some constructive suggestions which are very helpful to improve this manuscript. One of us, C. K. Chou would like to thank professor J. L. Han for his hospitality and encouragement. This work was supported in part by the National Natural Science Foundation of China under grants 11565020, 10773005, and the Counterpart Foundation of Sanya under grant 2016PT43, the Special Foundation of Science and Technology Cooperation for Advanced Academy and Regional of Sanya under grant 2016YD28, the Scientific Research Starting Foundation for 515 Talented Project of Hainan Tropical Ocean University under grant RHDRC201701, and the Natural Science Foundation of Hainan province under grant 114012. 


\section{References}

Aab, A., Abreu, P., Aglietta, M., Al Samarai, I., et al., 2016, Phys. Rev. D., 94, 082002

Abbott, B. P., Abbott, R., Abbott, T. D., et al., 2016, Phys. Rev. Lett., 116, 1102

Allen, C. W., Aller, Lawrence H., 1956, science., 123, 735

Bethe, H. et. al., 1990, Reviews of Modern Physics, 62, 801

Boehm, C., et al. 2004, Phys. Rev. Lett.,, 92, 101301

Buras., et al., 2003, Phys. Rev. Lett., 90, 241101

Colgate, S. A., and White, R. H., 1966, Astrophys. J. 143, 626

Callan, C., et al., 1983, Nucl. Phys., 212, 391

Casse., et al., 2004, ApJL, 602, L17

Cheng, et al., 2007, A\&A, 473, 351

Chiu, H. Y., \& Morrison, P., 1960, Phys. Rev. Lett., 5, 573

Connaughton, V., et al., 2016, arXiv:1602.03920

Dai, Z., Peng, Q. H., and Lu, T., 1995, ApJ., 440, 815

Dai, Z., et al., 2016, ApJ., 817, 132

Davies, J. H., \& Davies, D. R. 2010., Earth's surface heat flux. Solid Earth, 1, 5.

Dong, S., et al., 2016, Science, 351, 6270

Eatough, R., P., et al., 2013, Nature, 591, 391

Falcke, H., and Marko S. B., 2013, arXiv:1311.1841V1

Hooft, G., 1974, Nucl. Phys. B., 79, 276

Chiu, H, Y., and Morrison, P., 1960, Physical Review Letters, 5,573

Kn ödlseder, J., et al., 2003, Astron. Astrophys. 411, 457

Liebendoerfer, M., et al., 2004, arXiv:astro-ph/0405029

Luo, Z, Q., Liu, M, Q., and Peng, Q, H, 2008, 32, 253

Lyutikov, M. , 2016, arXiv:1602.07352

Letessier-Selvon, A., Stanev, T., 2011, RvMP, 83, 907

Liu, J. J., 2013, MNRAS., 433, 1108

Liu, J. J., 2014, MNRAS., 438, 930

Liu, J. J., \& Gu, W. M., 2016, ApJS, 224, 29

Liu, J. J., \& Liu, D. M., 2016, Ap\&SS, 361, 246

Liu, J. J., Peng, Q. H., Hao, L. H., Kang, X. P., Liu, D. M., 2017, RAA., 17, 107, arXiv:1707.03504

Ma, Z, Q., \& Tang, J. F., Physics Letters, 1983, 126, 310

Parker, E.,1970, ApJ., 160: 383

Polyakov, A. M., 1974, JETP Lett. 20, 194

Peng, Q. H., 2004, Nuclear Physics A, 738, 515

Peng, Q. H., Lie Z. and Wang, D.,1985, Scientia Sinica (Serries A), 970, 977

Peng, Q. H., Wang, D. and Lie, Z. , 1985, 30, 1056

Peng, Q. H., and Chou C., 2001, ApJ., 551, 23

Peng, Q. H., 2002, High Energy Physics and Nuclear Physics, 26, 104 (in Chinese)

Peng, Q. H., Liu, J, J, Chou, C. K., 2016, Ap\&SS., 361, 388

Peng, Q. H., 2016, EPJWC., 109, 03001

Peng, Q. H., 2016, Chinese Science Bulletin, 61, 2960 (in Chinese)

Rubakov, V., 1981, JETP Lett., 33, 644

Shapiro, S. L., \& Teukolsky, S. A., 1983, "Black Holes, White Dwarfs, and Neutron Stars., A Wiley-Interscience Publication

Schatten, K. H., 1970, Phys. Rev. D., 1, 2245

Schatten, K. H., 1983, Phys. Rev. D., 27, 1525

Shnir, Y., 2005, Phys. Rev. D., 72, 5016

't Hooft, G., 1974, Nuclear Physics B., 79, 276

Wang, D., Peng, Q. H., and Lie, Z., 1985, Chinese Science Bulletin, 30, 210
Wang, W. et al. 2006, A\&A, 446, 943

Wang, W. et al. 2005, MNRAS, 358, 263

Wilson, J. R., and Mayle, R. W., 1988, ASIB.,216, 731

Wilczek, F., 1982, Phys. Rev. Lett. 48, 1146

Woosley, S. E., and Weaver,T.A., 1986, Annu. Rev. Astron. Astrophysics., 24, 205

Woosley, S. E., 1991, Strong, weak, and electromagnetic interactions in nuclei, atoms, and astrophysics. AIP Conference Proceedings, 242, 121

Zhang, B., 2016, arXiv: 1602.04542

Zhang, B., 2016, arXiv: 1602.08086

Zhu, H. S., 2003, Text book of Astronomy, Higher Education Press: Bei-Jing (in Chinese) 